\title{
Ideals in subalgebras of the group algebras
}

by

LEONARD Y. H. YAP (Houston)

1. Introduction. Throughout this note $G$ denotes a locally compact Abelian group. Let $p$ be a real number such that $1 \leqslant p<\infty$, and let $L_{p}(G)$ denote the usual Lebesgue class with respect to $\lambda$, the Haar measure of $G$. Write $L_{1}(G) \cap L_{p}(G)$ as $A_{p}(G)$, and for $f \in A_{p}(G)$, define $\|f\| \|_{p}$ $=\|f\|_{1}+\|f\|_{p}$. It is easy (see (2.1) below) to verify that $A_{p}(G)$ is a Banach algebra with respect to $\|l \cdot \mid\|_{p}$ (multiplication in $A_{p}(G)$ is the usual convolution). It is plain that if $p=1$ (and $G$ is arbitrary) or $G$ is discrete (and $1 \leqslant p<\infty)$, then $A_{p}(G)$ is precisely the group algebra $L_{1}(G)$ and \|\|$\cdot\|\|_{p}$ is equivalent to $\|\cdot\|_{1}$; if $G$ is compact, then $A_{p}(G)=L_{p}(G)$ and $\|l \cdot\|_{p}$ is equivalent to $\|\cdot\|_{p}$. The purpose of this note is to present various properties of the algebra $A_{p}(G)$. Roughly speaking, our results say that some of the important known results of $L_{1}(G)$ can be extended to $A_{p}(G)$ while at the same time $A_{p}(G)$ lacks some of the useful properties possessed by $L_{1}(G)$. We have been motivated by the interesting papers of Porcelli-Collins [9, 10], Warner [15], and our earlier considerations of non-factorization theorems in [16]. The relationships of various results will be pointed out at the appropriate places.

Now we give a brief summary of the main results in the individual sections. In Section 2 we consider the factorization property of $A_{p}(G)$ (an algebra $A$ is said to have the factorization property if every element in $A$ can be written as $x \cdot y$ with $x, y$ in $A$ ) and it is proved that $A_{p}(G)$ has the factorization property if and only if $p=1$ or $G$ is discrete; $A_{p}(G)$ has a bounded approximate unit if and only if $p=1$ or $G$ is discrete. In Section 3 we prove that $A_{p}(G)$ is a regular semi-simple Banach algebra satisfying Ditkin's condition D (as defined in Loomis [7]) and the general Tauberian theorem for $A_{p}(G)$ : Let $I$ be a closed ideal in $A_{p}(G)$, then $I$ contains every element $f$ in kernel (hull $(I)$ ) such that the set [boundary $($ hull $(f)) \frown$ hull $(I)]$ contains no non-void perfect set. Warner [15] proves the results of Section 3 for $p=2$. In Section 4 we prove that 
(i) every maximal ideal in $A_{p}(G)$ is regular (closed) if and only if $p=1$ or $G$ is discrete;

(ii) every positive functional on $A_{p}(G)$ is continuous if and only if $p=1$ or $G$ is discrete;

(iii) it $I$ is a proper prime ideal in $A_{p}(G)$, then $I$ is regular maximal if and only if $I$ is closed; nite;

(iv) $A_{p}(G)$ contains a non-closed prime ideal if and only if $G$ is infi-

(v) every prime ideal of $A_{p}(G)$ is contained in a unique regular maximal ideal if and only if $G$ is discrete.

Thus the results in Section 4 are either extensions or "counterexamples" of the corresponding results in Porcelli-Collins $[9,10]$ and they answer the $A_{p}(G)$ version of the two questions (see [9]) raised at a recent international symposium held in Sopot, Poland. fact:

2. Factorization problems in $A_{p}(G)$. We begin with a very simple

(2.1) THEOREM. The function $\||\cdot|\|_{p}$ is a norm for the linear space $A_{p}(G)$ and $A_{p}(G)$ is a Banach algebra with respect to $\|\cdot \cdot\|_{p}$ if multiplication in $A_{p}(G)$ is the usual convolution of functions.

Proof. That $\||\cdot|\|_{p}$ is a norm is obvious, while completencss easily follows from the definition of $\||\cdot|\|_{p}$, the completeness of $L_{r}(G)(1 \leqslant r<\infty)$ and the fact: $\left\|f_{n}-f\right\|_{r} \rightarrow 0$ implies that $f_{n_{k}} \rightarrow f$ a.e. for some subsequence $\left(f_{n_{k}}\right)$ of $\left(f_{n}\right)$. Finally, to complete the proof, recall that $\|f * g\|_{p} \leqslant\|f\|_{1} \cdot\|g\|_{p}$ for all $f \in L_{\mathbf{1}}(G)$ and $g \in L_{p}(G)$. Using this fact we immediately have $\|f * g\|_{p}$ $\leqslant\|\| f\left\|_{p} \cdot\right\| g \|_{p}$ for all $f, g$ in $A_{p}(G)$.

For the convenience of the reader, we now review briefly what we need from the theory of $L(p, q)$-spaces.

(2.2) Definimion. Let $f$ be a complex-valued measurable function defined on $(G, \lambda)$, where $\lambda$ is the Haar measure of $G$. For $y \geqslant 0$, we define

$$
m(f, y)=\lambda\{x \in G:|f(x)|>y\} .
$$

Note that $m(f, \cdot)$ is a non-increasing, right-continuous function defined on $[0, \infty)$. For $x \geqslant 0$, we define

$$
\begin{aligned}
f^{*}(x) & =\inf \{y: y>0 \text { and } m(f, y) \leqslant x\} \\
& =\sup \{y: y>0 \text { and } m(f, y)>x\}
\end{aligned}
$$

with the conventions inf $\varnothing=\infty$ and $\sup \emptyset=0$. We note that $f^{*}$ is a non-increasing, right-continuous function. For $x>0$, we write

$$
f^{* *}(x)=\frac{1}{x} \int_{0}^{x} f^{*}(t) d t
$$

and let

$$
\|f\|_{(p, q)}=\left\{\int_{0}^{\infty}\left[x^{1 / p} f^{* *}(x)\right]^{q} \frac{d x}{x}\right\}^{1 / q}
$$

where $1<p<\infty, 1 \leqslant q<\infty$. We say that $f \in L(p, q)(G)$ if $\|f\|_{(p, q)}<\infty$. A theorem of Hardy (see [17], p. 20) shows that (the case $q=1$ is obtained by passing to the limit)

$$
\|f\|_{(p, q)} \leqslant p^{\prime}\left\{\int_{0}^{\infty}\left[x^{1 / p} f^{*}(x)\right]^{q} \frac{d x}{x}\right\}^{1 / q} \leqslant p^{\prime}\|f\|_{(p, q)},
$$

where $1 / p+1 / p^{\prime}=1$. Hardy's theorem ean also be used to show that $\|f\|_{p} \leqslant\|f\|_{(p, p)} \leqslant p^{\prime}\|f\|_{p}$, so that $L(p, p)=L_{p}$. The following fact (which is a special case of $([8],(2,6))$ will be useful to us later. A simple proof is given in $[16],(2.2)$.

(2.3) Theorem. If $p, r, s$ are real numbers such that $1<r, s<\infty$, $1 / r+1 / s>1$ and $1 / p=1 / r+1 / s-1$, then

$$
L_{r}(G) * L_{s}(G) \subseteq L(p, 1)(G) .
$$

Recall that an algebra $A$ is said to have the factorization property if $A \cdot A=A$. $A \cdot A$ will be written as $A^{2}$ throughout the rest of this note.

(2.4) THEOREM. The Banach algebra $A_{p}(G)$ has the factorization property $\Leftrightarrow p=1$ (and $G$ is arbitrary) or $G$ is discrete (and $1 \leqslant p<\infty$ ).

Proof. The implication $\Leftarrow$ is the well-known factorization theorem of P.J. Cohen (see, for example, Cohen [1], Hewitt [4], Koosis [6]).

Next we suppose that $1<p<\infty$ and $G$ is non-discrete. We will construct a function $F$ in $A_{p}(G)$ such that $F$ is not in $\left(A_{p}(G)\right)^{2}$. Define $r=s=2 p /(1+p)$ so that $1<r=s<\infty, r<p$, and $1 / p=1 / r+1 / s-1$. By (2.3), we have

$$
\left(A_{p}(G)\right)^{2} \subseteq A_{r}(G) * A_{s}(G) \subseteq L(p, 1)(G) .
$$

Thus it suffices to define a function $F$ in $A_{p}(G)$ such that $F$ is not in $L(p, 1)(G)$. Define $\beta=1 /(2 p)+1 / 2$, and observe that $0<\beta<1$ and $\beta p>1$. Next we choose a positive integer $n_{0}$ such that $n_{0}>e^{p \beta}$ and then choose a sequence $\left(\nabla_{n}\right)_{n=n_{0}}^{\infty}$ of pairwise disjoint Haar measurable subsets of $G$ such that

$$
\lambda\left(\nabla_{n}\right)=\frac{1}{n(n+1)}, \quad n=n_{0}, n_{0}+1, \ldots
$$

Write

$$
F=\sum_{n=n_{0}}^{\infty} a_{n} \xi_{\nabla_{n}}
$$


where $a_{n}=n^{\mathrm{i} / p}(\log n)^{-\beta}$, and $\xi_{E}$ denotes the characteristic function of $E$. It is clear that $F \in A_{p}(G)$ and it remains to show that

$$
\int_{0}^{\infty}\left[x^{1 / p} F^{*}(x)\right] \frac{d x}{x}=\infty .
$$

But straight-forward computations show that

$$
m(F, y)=\left\{\begin{array}{cl}
1 / n_{0}, & \text { if } 0 \leqslant y<a_{n_{0}}, \\
1 /(n+1), & \text { if } a_{n} \leqslant y<a_{n+1},
\end{array}\right.
$$

$$
F^{*}(x)=a_{n} \quad \text { if } 1 /(n+1) \leqslant x<1 / n \text {. }
$$

Finally, an easy calculation (using (ii) and $\sum n^{-1}(\log n)^{-\beta}=\infty$ ) gives (i) immediately.

(2.5) Corollary. The Banach algebra $A_{p}(G)$ has a bounded approximate unit $\Leftrightarrow p=1$ or $G$ is discrete.

Proof. The implication $\Leftarrow$ is of course well-known. The implication $\Rightarrow$ immediately follows from the preceding theorem and Hewitt's factorization theorem [4]: let $A$ be a Banach algebra and let $V$ be a Banach $A$-module (that is, $V$ is an $A$-module in the algebraic sense and $\|a v\| \leqslant\|a\| \cdot\|v\|$ for all $a \in A, v \in V)$ and suppose that there is a constant $M>0$ such that for $a \in A, v \in V$ and $\varepsilon>0$, there exists $e \in A$ such that $\|e\| \leqslant M,\|a-a e\|<\varepsilon$ and $\|v-e v\|<\varepsilon$. Then $A \cdot V=V$.

(2.6) Remark. Hewitt's factorization theorem implies that $I_{1}(G) * A_{p}(G)=A_{p}(G)$ for all $G$ and $1 \leqslant p<\infty$. It is easy to verify that if $A$ and $V$ are as in Hewitt's theorem and suppose that $A_{0}$ and $V_{0}$ are dense subsets of $A$ and $V$, respectively, then $A_{0} \cdot V_{0}$ is dense in $A \cdot V=V$. Hence $\left(C_{00}(G)\right)^{2}$ is dense in $A_{p}(G)$, where $O_{00}(G)$ denotes the set of all continuous functions defined on $G$ with compact supports.

We need the following corollary in Section 4:

(2.7) Corollary. If $1<p<\infty$ and $G$ is non-discrete, then $\left(A_{p}(G)\right)^{2}$ is a dense proper subset of $A_{p}(G)$.

The situation for unbounded approximate unit is much more pleasant. Let $\mathscr{V}$ denote the family of all precompact neighborhoods of 0 , the identity element of $G$. Partially order $\mathscr{V}$ by set inclusion and denote it by $\left\{V_{d}\right\}$. Then $\left\{V_{d}\right\}$ is a directed family, and for each $V_{d}$, choose a nonnegative continuous function $v_{d}$ with support $\left(v_{d}\right) \subset V_{d}$ and $\int v_{d} d \lambda=1$. Then we have (see Loomis [7], p. 124) the following which is needed in the next section:

(2.8) THEORem. The net $\left\{v_{d}\right\}$ is an (probably unbounded) approximate unit for $A_{p}(G)$.
(2.9) Remarks. (a) We will have occasions to apply the above results in Section 4 to obtain both "positive" and "negative" theorems related to those in Porcelli-Collins $[9,10]$.

(b) The usefulness of bounded approximate units is of course well known and many interesting theorems have been obtained for algebras with bounded approximate units. For more recent examples, see Varopoulos [14], Rieffel [12], and Porcelli-Collins [10], Theorem 1.

3. Tauberian theorems for $A_{p}(G)$. We continue to use $G$ to denote a locally compact Abelian group with character group $\Gamma$. The Haar measure of $\Gamma$ is denoted by $\mu$. All terms and notation not explained here are as in Loomis [7].

(3.1) THEOREM. The maximal ideal space $M_{p}(G)$ of the commutative Banach algebra $A_{p}(G)$ can be identified with $\Gamma$.

Proof. For $0 \neq f \in A_{p}(G)$, we have

$$
\|\| f^{n}\left|\left\|_{p} \leqslant\right\| f^{n-1}\left\|_{1} \cdot\right\| f\left\|_{1}+\right\| f^{n-1}\left\|_{1}\right\| f\left\|_{p} \leqslant\right\| f\left\|_{1}^{n-1} \mid\right\| f\|\|_{p}\right.
$$

Hence

$$
\lim _{n \rightarrow \infty}\|\| f^{n}\|\|_{p}^{1 / n} \leqslant \lim _{n \rightarrow \infty}\|f\|_{1}^{(n-1) / n} \cdot\|\| f\left\|_{p}^{1 / n} \leqslant\right\| f \|_{1} .
$$

Therefore

$$
\lim \left\|f^{n}\right\|_{p}^{1 / n} \leqslant\|f\|_{1} \quad \text { for all } f \epsilon A_{p}(G) .
$$

Now if $\gamma \in M_{p}(G)$, then

$$
|\gamma(f)|^{n}=\left|\gamma\left(f^{n}\right)\right| \leqslant\left\|\mid f^{n}\right\| \|_{p}
$$

Hence

$$
|\gamma(f)| \leqslant \lim _{n \rightarrow \infty}\left\|\mid f^{n}\right\|\left\|_{p}^{1 / n} \leqslant\right\| f \|_{1},
$$

and so $\gamma$ is $\|\cdot\|_{1}$-bounded and hence can be extended in a unique fashion to a multiplicative linear functional $\gamma_{1}$ on $L_{1}(G)$ and, conversely, every multiplicative linear functional $\gamma_{1}$ on $L_{1}(G)$ determines a $\gamma \in M_{p}(G)$. Now recall that the maximal ideal space of $L_{1}(G)$ is $\Gamma$ and observe that the Gelfand topology on $M_{p}(G)$ agrees with the usual topology of $\Gamma$.

The next two lemmas are extensions of (and will be substitutes for) the corresponding results in Rudin [13], 2.6.1 and 2.6.2, and will play the same role.

(3.2) LeMma. Suppose $C$ is a compact subset of $\Gamma, V \subset \Gamma$, and $0<\mu(\nabla)<\infty, \mu(C-V)<\infty$, where $\mu$ is the Haar measure of $\Gamma$. Then there exists $k \in A_{p}(G)$ such that

(a) $\hat{k} \equiv 1$ on $C, \hat{k} \equiv 0$ outside of $C+\nabla-\nabla$ and $0 \leqslant \hat{k} \leqslant 1$;

(b) \|\|$k \|_{p} \leqslant\left(\mu(C-\nabla) /\left.\mu(V)\right|^{1 / 2}+\left(\mu(C-V)^{1-1 / 2 p}\right) / \mu(\nabla)^{1 /(2 p)}\right.$. 
Proof. Let $g$ and $h$ be the inverse Plancherel transforms of the characteristic functions of $V$ and $C-V$, respectively, and define

$$
k(x)=\mu(V)^{-1} g(x) h(x) \quad(x \in G) .
$$

Now by Rudin [13], p. 49, we see that $k \in L_{1}(G)$ and it satisfies condition (a), as well as

$$
\|k\|_{1} \leqslant(\mu(C-V) / \mu(V))^{1 / 2} .
$$

But $k$ is also in $L_{p}(G)$ (because $\hat{k}=\mu(V)^{-1} \hat{g} * \hat{h}$; see Edwards [3], 10.4.7, if necessary) and

$$
\begin{aligned}
\|k\|_{p} & =\mu(V)^{-1}\|g h\|_{p}=\mu(V)^{-1}\|g h\|_{\infty}^{1-1 / p}\|g h\|_{1}^{1 / p} \\
& \leqslant \mu(V)^{-1}\left(\|g\|_{\infty}\|h\|_{\infty}\right)^{1-1 / p}\|g\|_{2}^{1 / p}\|h\|_{2}^{1 / p} \\
& \leqslant \mu(V)^{-1}\left(\|\hat{g}\|_{1}\|\hat{h}\|_{1}\right)^{1-1 / p}\|g\|_{2}^{1 / p} \cdot\|h\|_{2}^{1 / p} \\
& \leqslant \mu(C-V)^{1-1 / 2 p} \cdot \mu(V)^{-1 / 2 p} .
\end{aligned}
$$

(3.3) THEOREM. If $W$ is an open set in $\Gamma$ which contains a compact set $C$, then there exists $f$ in $A_{p}(G)$ such that $\hat{f} \equiv 1$ on $C$ and $\hat{f} \equiv 0$ outside of $W$.

Proof. Choose a neighborhood $V$ of 0 in $\Gamma$ such that $C+V-V \subset W$, $\mu_{0}(C-V)<\infty$ and then apply (3.2).

(3.4) Corollary. The commutative Banach algebra $A_{p}(G)$ is a regular semi-simple Banach algebra.

Proof. Immediate from (3.3) and Loomis [7], p. 57.

The main result in this section is the following Tauberian theorem. Some applications of this theorem will appear in the next section.

(3.5) TaUberian Theorem ( $\left.{ }^{1}\right)$. Let $I$ be a closed ideal in $A_{p}(G)$. Then $I$ contains every element $f$ in kernel $(h u l l(I))$ such that the intersection of the boundary of hull $(f)$ with hull $(I)$ contains no non-void perfect set.

In view of Theorem $25 \mathrm{~F}$ in Loomis [7], p. 86, and Corollary (3.4), it suffices to show that the algebra $A_{p}(G)$ satisfies Ditkin's condition D: If $f \in A_{p}(G)$ and $\gamma \in \Gamma$ such that $f(\gamma)=0$, then there exists a sequence $\left(f_{n}\right)$ in $A_{p}(G)$ such that $\hat{f}_{n} \equiv 0$ on some neighborhood $V_{n}$ of $\gamma$ and \|\|$f * f_{n}-f \|_{p} \rightarrow 0$; if $\Gamma$ is non-compact, the condition must also be satisfied for the point at infinity, that is, for each $f$ in $A_{p}(G)$, there exists a sequence $\left(f_{n}\right)$ in $A_{p}(G)$ such that $\hat{f}_{n}$ has compact support and $\left\|f * f_{n}-f\right\|_{p} \rightarrow 0$. The case $p=1$ is of course well known (see, for example, [7]) and the standard proof makes use of the bounded approximate units in $A_{1}(G)$. Warner [15] has proved it for $p=2$ and also observed (in our notation) that

(1) Professor Edwin Hewitt has informed me (November 1968) that he and Professor K.A. Ross have also obtained (jointly) this theorem.
$A_{p}(G)$ lacks a bounded approximate unit if $G$ is neither compact nor discrete and $1<p \leqslant 2$. The following lemmas, which lead to a proof that $A_{p}(G)$ satisfies Ditkin's condition D, are modifications of the corresponding lemmas in Warner's paper. We have included all the details for the convenience of the reader.

(3.6) Lemma. The algebra $A_{p}(G)$ satisfies Ditkin's condition $D$ at infinity.

Proof. It suffices to show that for $0 \neq f \in A_{p}(G)$ and $\varepsilon>0$, there exists a function $g \in A_{p}(G)$ such that $\hat{g}$ has compact support and \|\|$g * f-f\|\|_{p}$ $<\varepsilon$. First choose a function $h$ (from a possibly unbounded approximate unit (see (2.8)) in $A_{p}(G)$ such that

$$
\|f * h-f\|_{p}<\varepsilon / 2 .
$$

Now by 2.6 .6 of Rudin [13], there exists a $k$ in $L_{1}(G)$ such that $\hat{k}$ has compact 'support and

$$
\|h * k-h\|_{1}<\varepsilon / 2\|\| f \|_{p} .
$$

Note that $g=h * k$ is in $A_{p}(G)$ and since $g * f-f=f *(g-h)+f * h-f$, we obtain $\|\mid g * f-f\|_{p}<\varepsilon$ and note that $\hat{g}=\hat{h} \hat{k}$ has compact support.

Let $\mathscr{U}=\left\{U_{\lambda}\right\}_{\lambda \in A}$ be the family of all symmetric neighborhoods of the identity element 0 in $\Gamma$ of measure $\leqslant 1$. Then $\mathscr{U}$ is a directed family under set inclusion. Let $\left\{V_{\lambda}\right\}_{\lambda \in A}$ denote any net of symmetric precompact neighborhoods of 0 satisfying the following conditions:

(i) given $U_{\lambda}$ in $\mathscr{U}, \bar{V}_{\lambda} \subseteq U_{\lambda}$ and $\mu\left(U_{2}\right)<4 \mu\left(V_{\lambda}\right)$ ( $\mu$ is the Haar measure on $\Gamma$ );

(ii) given $U_{\lambda} \epsilon \mathscr{U}$ and $V_{\lambda}$, there is a neighborhood $W_{\lambda}$ of 0 such that $\nabla_{\lambda}+W_{\lambda} \subseteq U_{\lambda}$.

(3.7) LeMMA. There exists a net $\left(k_{\lambda}\right)_{\lambda \in A}$ in $A_{p}(G)$ such that, for each $\lambda \in \Lambda$, we have (a) $\left\|\mid k_{\lambda}\right\| \leqslant 6$; (b) $k_{\lambda} \equiv 1$ on some neighborhood $W_{\lambda}$ of 0 .

Proof. For given $U_{\lambda}$ in $\mathscr{U}$, let $V_{\lambda}$ be the corresponding set as above. Let $g_{\lambda}, h_{\lambda}$ be the inverse Plancherel transforms of the characteristic functions of $U_{\lambda}$ and $V_{\lambda}$, respectively. Defining $k_{\lambda}=\mu_{0}\left(V_{\lambda}\right)^{-1} g_{\lambda} h_{\lambda}$ and comparing $k_{2}$ with the function $k$ defined in the proof of (3.2), we see (by (3.2.(b))) that $\left\|\mid k_{\lambda}\right\| \|_{p} \leqslant 6$. To show (b); let $W_{\lambda}$ be the neighborhood corresponding to $U_{\lambda}$ and $V_{\lambda}$ as described in (ii) above, and then use $\hat{k}_{\lambda}=\mu\left(V_{\lambda}\right)^{-1} \hat{g}_{\lambda} * \hat{h}_{\lambda}$ to see that $k_{\lambda} \equiv 1$ on $W_{\lambda}$.

(3.8) Lemma. For any compact set $C \subset G$ and $\varepsilon>0$, there exists $\lambda_{0}$ in $A$ such that if $k$ is in $\left\{k_{\lambda} \mid \lambda>\lambda_{0}\right\}$, then $\left\|\mid k-k_{s}\right\|_{p}<\varepsilon$ for every $s \in C$.

Proof. Recall that the set $U(C, \delta)=\{\gamma \in \Gamma||(x, \gamma)-1 \mid<\delta$ for all $x \in C\}$ is a neighborhood of 0 in $\Gamma(\delta>0)$. Hence there exists a $\lambda_{0}$ such 
that if $\lambda>\lambda_{0}$, then $U_{\lambda}^{2} \subseteq U(C, \delta)$, where $\delta=\min \left(\varepsilon / 4,(\varepsilon / 4)^{p}\right)$. Now let $k \in\left\{k_{\lambda} \mid \lambda>\lambda_{0}\right\}$ and suppose $k=\mu(V)^{-1} g h$, where $g, h, V$ correspond to $g_{\lambda}, h_{\lambda}, V_{\lambda}$, respectively, in the proof of the preceding lemma. It follows that $\hat{k} \equiv 0$ outside of $U V$. It remains to show that $\left\|\left|k-k_{s}\right|\right\|_{p}<\varepsilon$ for every $s \epsilon C$. Observe that

$$
\left\|k-k_{s}\right\|_{2}^{2}=\left\|\hat{k}_{-}-\hat{k}_{s}\right\|_{2}^{2}=\int_{U V}|k(\gamma)|^{2}|1-(s, \gamma)|^{2} d \gamma<\delta^{2} .
$$

Thus $\left\|k-k_{s}\right\|_{2}<\delta$ and similarly $\left\|g-g_{s}\right\|_{2}<\delta \mu(U)^{1 / 2},\left\|h-h_{s}\right\|_{2}<\delta \mu(V)^{1 / 2}$. Hence

$$
\begin{aligned}
\left\|k-k_{s}\right\|_{1} & \leqslant \mu(V)^{-1}\left\{\left\|g\left(h-h_{s}\right)\right\|_{1}+\left\|h_{s}\left(g-g_{s}\right)\right\|_{1}\right\} \\
& \leqslant \mu(V)^{-1}\left\{\|g\|_{2}\left\|h-h_{s}\right\|_{2}+\left\|h_{s}\right\|_{2}\left\|g-g_{s}\right\|_{2}\right\} \\
& \leqslant \delta(\mu(U) / \mu(V))^{1 / 2}<2 \delta .
\end{aligned}
$$

Next we compute $\left\|k-k_{s}\right\|_{p}$ for $s \epsilon C$. We have

$$
\begin{aligned}
\left\|k-k_{s}\right\|_{p}^{p} & \leqslant\left\|k-k_{i}\right\|_{\infty}^{p-1} \cdot\left\|k-k_{s}\right\|_{1} \leqslant 2^{p-1}\left\|k_{n}\right\|_{\infty}^{p-1} \cdot 2 \delta \\
& \leqslant 2^{p} \delta\|\hat{k}\|_{1}^{p-1}=2^{p} \delta,
\end{aligned}
$$

since $\hat{k}=\mu(V)^{-1} \xi_{U} * \xi_{V}$ and the $L_{1}$-norm of $\xi_{U} * \xi_{V}$ is $\mu(U) \cdot \mu(V)$. Finally,

$$
\left\|\left|k-k_{s}\right|\right\|_{p} \leqslant 2 \delta+2 \delta^{1 / p}<2 \cdot(\varepsilon / 4)+2(\varepsilon / 4)=\varepsilon .
$$

(3.9) Corollart. If $f \in A_{p}(G)$ and $\hat{f}(0)=0$, then $\lim \left|\left\|f * k_{\lambda} \mid\right\|_{p}=0\right.$. Proof. Let $\delta>0$ be given. Choose symmetrie compact $C \subseteq G$ such that

$$
\int_{C^{\prime}}|f(x)| d x<\frac{\delta}{24} .
$$

Put $\varepsilon=\delta / 3\|f\|_{1}$ and choose $\lambda_{0}$ so that if $k$ is in $\left\{k_{\lambda} \mid \lambda>\lambda_{0}\right\}$, then \|\|$k-k_{s} \|_{p}<\varepsilon$ for $s \epsilon C$. Hence

Therefore

$$
\begin{aligned}
(f * k)(t) & =\int f(s) k(t-s) d s-\hat{f}(0) k(t) \\
& =\int f(s)\left[k_{-s}(t)-k(t)\right] d s .
\end{aligned}
$$

$$
\begin{aligned}
\|f * k\|_{p} & =\left\{\int_{G}\left|\int_{G} f(s)\left[k_{-s}(t)-k(t)\right] d s\right|^{p} d t\right\}^{1 / p} \\
& \leqslant \int_{G}\left\{\int_{G}\left|f(s)\left[k_{-s}(t)-k^{\prime}(t)\right]\right|^{p} d t\right\}^{1 / p} d s
\end{aligned}
$$$$
=\int_{G}|f(s)| \cdot\left\|k_{-s}-k_{i}\right\|_{p} d s
$$

(by [2], p. 530)

Similarly,

$$
\|f * k\|_{1} \leqslant \int_{G}|f(s)| \cdot\left\|k_{-s}-k\right\|_{1} d s .
$$

Therefore

$(*)$

$$
\left\|| | f * k \left|\left\|_{p} \leqslant \int_{C}|f(s)| \cdot\left|\left\|k_{-s}-k\left|\left\|_{p} d s+\int_{C^{\prime}}|f(s)| \cdot\right\|\right|\left|k_{-s}-k\right|\right\|_{p} d s .\right.\right.\right.\right.
$$

Now if $s \epsilon C$, then $\left\|\left|k_{-s}-k\right|\right\|_{\eta}<\varepsilon$, therefore $(*)$ implies

$$
\|\| f * k\left|\left\|_{p}<\varepsilon\right\| f\left\|_{1}+2\right\|\|k \mid\|_{p} \cdot \delta / 24<\delta .\right.
$$

(3.10) TheOREM, $A_{p}(G)$ satisfies Dittin's condition $\mathbf{D}$ at all (finite) points.

Proof. It suffices to show that $A_{p}(G)$ satisfies condition $\mathrm{D}$ at the identity element 0 of $\Gamma$. Let $\left\{v_{a}\right\}$ be the (probably unbounded) approximate unit described in (2.8) and let $\left(k_{\lambda}\right)_{\lambda \in \Lambda}$ be as in Lemma (3.7). Write

$$
v_{(a, \lambda)}=v_{d}-k_{\lambda} * v_{d} \text {. }
$$

Clearly, $v_{(a, \lambda)}$ is in $A_{p}(G)$. Now we order the pairs $(d, \lambda)$ as follows: $\left(d_{1}, \lambda_{1}\right)>\left(d_{2}, \lambda_{2}\right) \Leftrightarrow d_{1}>d_{2}$ and $\lambda_{1}>\lambda_{2}$. Now let $q$ run through this directed set; then $\left(v_{q}\right)$ is a net and $\hat{v}_{q}=\hat{v}_{d}-\hat{k}_{\lambda} \hat{v}_{d}=\hat{v}_{d}\left(1-\hat{k}_{\lambda}\right)$ which is identically zero on some neighborhood $W_{\lambda}$ of 0 (see condition (b) in (3.7)). Finally for $f \in A_{p}(G)$ and $\hat{f}(0)=0$, we have

$$
\lim \|\| v_{q} * f-f\|\|_{p} \leqslant \lim \left(\left\|\left|v_{d} * f-f\right|\right\|_{p}+\left\|v_{d}\right\|_{1} \cdot\|\| k_{\lambda} * f \mid \|_{p}\right)=\mathbf{0} .
$$

This completes the proof that $A_{p}(G)$ satisfies condition $\mathrm{D}$ and also the proof of the Tauberian Theorem (3.5).

We record two corollaries below for later application.

(3.11) Corollart. If $I$ is a closed ideal in $A_{p}(G)$ and if hull $(I)$ is empty, then $I=A_{p}(G)$.

(3.12) CoRoltaRY. Every proper closed ideal in $A_{p}(G)$ is contained in a regular maximal ideal.

4. Maximal, regular, and prime ideals in $A_{p}(G)$. Throughout this section, $G$ will continue to denote a locally compact Abelian group with character group $\Gamma$. The purpose of this section is either to extend or to show the impossibility of extending the theorems in Porcelli-Collins $[9,10]$ from $L_{1}(G)$ to $A_{p}(G)$. We begin by recalling some facts that will be needed later. Let $\left[R^{2}\right]$ denote the ideal generated by $R^{2}$ in the sequel.

(4.1) THEOREM ([11], p. 88). (a) If $R$ is a commutative Banach algebra such that $\left[R^{2}\right] \neq\{0\}$, then $R$ contains a non-prime maximal ideal $\Leftrightarrow\left[R^{2}\right] \ddagger R$. Each non-prime maximal ideal is a maximal subspace of $R$ which contains $\left[R^{2}\right]$.

(b) If $R$ is a commutative Banach algebra without identity and $M$ is a maximal ideal in $R$, then $M$ is regular $\Leftrightarrow M$ is prime.

(4.2) THeorem. Every maximal ideal in $A_{p}(G)$ is regular $\Leftrightarrow p=1$ or $G$ is discrete. 
Proof. The implication $<$ is proved in [10], Theorem 1 , while $\Rightarrow$ immediately follows from the proof of (2.4) and (4.1).

(4.3) Corollary. Every maximal ideal in $A_{p}(G)$ is closed $\Leftrightarrow p=1$ or $G$ is discrete.

Proof. The implication $\Leftarrow$ is proved in [10], Corollary 1 , while $\Rightarrow$ follows from the last assertion in (4.1(a)), (4.2) and (2.7).

(4.4) Corollary. Every positive functional on $A_{p}(G)$ is continuous $\Leftrightarrow p=1$ or $G$ is discrete.

(A linear functional $F$ defined on a *algebra is positive if $F\left(x x^{*}\right) \geqslant 0$ for all $x \in A$.)

Proof. The implication $\Leftarrow$ is well-known result of Varopoulos [14]. Now suppose $p>1$ and $G$ is non-discrete. By (4.3) there is a maximal non-closed ideal $M$ in $A_{p}(G)$ such that $\left[\left(A_{p}(G)\right)^{2}\right] \subseteq M$. Thus there exists $f_{0} \in A_{p}(G)$ such that $A_{p}(G)=M+\left\{\alpha f_{0}\right\}$. Define $F$ by $F\left(m+\alpha f_{0}\right)=\alpha$; then $F$ is a positive linear functional, but it is not continuous.

(4.5) Leirma. If $I$ is an ideal in $A_{p}(G)$ such that $I$ is contained in exactly one regutar maximal ideal, say $M$, then $\bar{I}=M$.

Proof. Let $\gamma$ be the character in $\Gamma$ corresponding to $M$. Thus hull $(\bar{I})$ $=\{\gamma\}$ and by the Tauberian theorem (3.5) we have $\bar{I} \doteq M$.

(4.6) Lemma. If a prime ideal $I$ of $A_{p}(G)$ is contained in a regular maximal ideal, then $I$ is contained in only one regular maximal ideal.

Pro of. Same proof as Lemma 2 in [10], use our Theorem (3.3) instead of [13], 2.6.2.

(4.7) LEMma. If $I$ is an ideal in $A_{p}(G)$ such that $I$ is contained in no regular maximal ideal, then $\bar{I}=A_{p}(G)$.

Proof. It follows from (3.5).

(4.8) THEOREM. If $I$ is a proper prime ideal in $A_{p}(G)$, then $I$ is regular maximal $\Leftrightarrow I$ is closed.

Proof. Consider the implication $\leftarrow$. By (3.12), $I$ is contained in a regular maximal ideal $M$. Hence $I=M$ by (4.6) and (4.5). The converse is of course valid for all Banach algebras. infinite.

(4.9) Theorem. $A_{p}(G)$ contains a non-closed prime ideal $\Leftrightarrow G$ is

Proof. Only $\Leftarrow$ requires proof. We consider two cases:

(a) If $G$ is discrete, then $A_{p}(G)=L_{1}(G)$ and we are done by Theorem 3 of $[10]$.

(b) If $G$ is non-discrete so that $\Gamma$ is non-compact. Now argue as in the first half of the proof of Theorem 3 in [10] (apply (4.7) and (3.3) at the appropriate points).
(4.9) THEOREM. Every prime ideal of $A_{p}(G)$ is contained in a unique regular maximal ideal $\Leftrightarrow G$ is discrete.

Proof. Similar to the proof of Theorem 4 in [10].

Added in proof. I wish to thank Professor Edwin Hewitt for informing me that some of our results in Section 3 overlap certain results in Hans Reiter's new monograph: Classical harmonic analysis and locally compact groups. Reiter deals with a large class of subalgebras of $L_{1}(G)$ called Segal algebras, of which the algebras $A_{p}(G)$ are examples. We have been able to prove that the Tauberian theorem (3.5) is valid for all Segal algebras.

\section{References}

[1] P. J. Cohen, Factorization in group algebras, Duke Math. J. 26 (1959), p. 199-205. [2] N. D unford and J. T. Schwartz, Linear operators, Part I, New York 1958.

[3] R. E. Edwards, Functional analysis, New York 1965.

[4] E. Hewitt, The ranges of certain convolution operators, Math. Seand. 15 (1964), p. 147-155.

[5] - and K. A. Ross, Abstract harmonic analysis I, Berlin 1963.

[6] P. Koosis, Sur un théorème de Paul Cohen, C. R. Acad. Sc. Paris 259 (1964), p. 1380-1382.

[7] L. H. Loomis, An introduction to abstract harmonic analysis, Princeton New Jersey 1953.

[8] R. O'N eil, Oonvolution operators and $L(p, q)$-spaces, Duke Math. J. 30 (1963), p. 129-142.

[9] P. Porcelli and H. S. Collins, Ideals in grou palgebras, Bull. Amer. Math. Soc. 75 (1969), p. 83-84.

[10] - Ideals in group algebras, Studia Math. 33 (1969) p. 223-226.

[11] P. Porcelli, Linear spaces of analytic functions, New York 1966.

[12] M. A. Rieffel, On the continuity of certain intertwining operators, centralizers, and positive linear functionals, Proc. Amer. Math. Soc. 20 (1969), p. 455-457.

[13] W. Rudin, Fourier analysis on groups, New York 1962.

[14] N. T. Varopoulos, Sur les formes positive d'une algèbre de Banach, C. R. Acad. Sc. Paris 258 (1964), p. 2465-2467.

[15] C. R. Warner, Closed ideals in the group algebra $L^{1}(G) \cap L^{2}(G)$, Trans. Amer. Math. Soc. 121 (1966), p. 408-423.

[16] L. Y. H. Y ap, On the impossibility of representing certain functions by convolutions, Math. Scand. 24 (1969).

[17] A. Zygmund, Trigonometric series, Vol. I, 2nd ed., Cambridge 1959

Reçu par la Rédaction le 23. 4. 1969 